\title{
Algebraic approach to quantum field theory on non-globally-hyperbolic spacetimes
}

\author{
Ulvi Yurtsever \\ Department of Physics \\ University of California \\ Santa Barbara, CA 93106
}

October, 1992

UCSBTH-92-43

\begin{abstract}
The mathematical formalism for linear quantum field theory on curved spacetime depends in an essential way on the assumption of global hyperbolicity. Physically, what lie at the foundation of any formalism for quantization in curved spacetime are the canonical commutation relations, imposed on the field operators evaluated at a global Cauchy surface. In the algebraic formulation of linear quantum field theory, the canonical commutation relations are restated in terms of a welldefined symplectic structure on the space of smooth solutions, and the local field algebra is constructed as the Weyl algebra associated to this symplectic vector space. When spacetime is not globally hyperbolic, e.g. when it contains naked singularities or closed timelike curves,
\end{abstract}


a global Cauchy surface does not exist, and there is no obvious way to formulate the canonical commutation relations, hence no obvious way to construct the field algebra. In a paper submitted elsewhere, we report on a generalization of the algebraic framework for quantum field theory to arbitrary topological spaces which do not necessarily have a spacetime metric defined on them at the outset. Taking this generalization as a starting point, in this paper we give a prescription for constructing the field algebra of a (massless or massive) KleinGordon field on an arbitrary background spacetime. When spacetime is globally hyperbolic, the theory defined by our construction coincides with the ordinary Klein-Gordon field theory on a globally hyperbolic background. We explore some basic features of our generalized KleinGordon theory on arbitrary spacetimes, and study its specific properties on simple examples of non-globally-hyperbolic backgrounds that contain closed timelike curves or naked singularities. 


\section{The algebraic approach to quantum field theory}

The quantum theory of a linear Klein-Gordon field on a globally hyperbolic spacetime $(M, g)$ can be built entirely on the basis of the well known canonical commutation relations (CCR)

$$
\begin{aligned}
& {\left[\phi(x), \phi\left(x^{\prime}\right)\right]=0,} \\
& {\left[\phi(x), \dot{\phi}\left(x^{\prime}\right)\right]=i \delta\left(x, x^{\prime}\right), \quad \text { for } x, x^{\prime} \in \Sigma,}
\end{aligned}
$$

where $\phi(x)$ denotes the field operator "evaluated" at the point $x, \Sigma$ is a Cauchy surface for spacetime, and $\dot{\phi}$ denotes the normal time derivative of the field evaluated on $\Sigma$. The CCR provide well-posed initial data on $\Sigma$ for the antisymmetric two-point function $\left[\phi(x), \phi\left(x^{\prime}\right)\right]$, which can then be evolved via the Klein-Gordon field equation

$$
\left(\nabla^{a} \nabla_{a}-m^{2}\right) \phi=0
$$

to yield the complete solution. It is also a consequence of the field equation (2) that all commutators on $\Sigma$ involving polynomial functions of the field $\phi$ and its normal time derivative ("canonical momentum") $\pi=\dot{\phi}$ can be determined solely from Eqs. (1).

Everyone knows, of course, that the field operator $\phi$ is ill-defined when evaluated at a point. To construct a mathematically well-defined quantum field theory, we need to work with the smeared field operators

$$
\phi(f) \equiv \int_{M} f(x) \phi(x) d \tau,
$$

where $f$ is a real-valued $C^{\infty}$ test function of compact support on $M$, and $d \tau$ denotes the spacetime four-volume form of $(M, g)$. To express the CCR [Eqs. (1)] in terms of these smeared field operators, we introduce the vec-

tor space, $S_{0}(M, g)$, which consists of the $C^{\infty}$ solutions of Eq. (2) that are compact-supported on $\Sigma$ (and hence are compact-supported on any Cauchy surface). On $S_{0}(M, g)$, there exists a natural, antisymmetric bilinear form (the Klein-Gordon inner product)

$$
\sigma(F, G) \equiv \int_{\Sigma}\left(F \nabla^{a} G-G \nabla^{a} F\right) d \Sigma_{a}, \quad F, G \in S_{0}(M),
$$

where $d \Sigma_{a}$ is the three-volume form of $\Sigma$. The integral in Eq. (4) exists because $F$ and $G$ are compact supported on $\Sigma$, and it is independent of the choice of Cauchy surface $\Sigma$ because $F$ and $G$ satisfy the wave equation (2). 
The Klein-Gordon form $\sigma$ makes $S_{0}(M)$ into a symplectic vector space. To connect this symplectic structure with the CCR, we let $C_{0}^{\infty}(M)$ denote the space of smooth test functions of compact support on $M$. It follows [1] from the global hyperbolicity of $(M, g)$ that for each $f \in C_{0}^{\infty}(M)$ there exists a unique solution, $\mathcal{E}^{+} f$, of the inhomogeneous wave equation

$$
\left(\nabla^{a} \nabla_{a}-m^{2}\right) \phi=f
$$

that vanishes outside $J^{-}(\operatorname{support}(f))$, called the "advanced" solution with source $f$, and a unique solution, $\mathcal{E}^{-} f$, that vanishes outside $J^{+}(\operatorname{support}(f))$, called the "retarded" solution. Moreover, the advanced and retarded solutions $\mathcal{E}^{ \pm} f$ are compact supported on Cauchy surfaces since $f$ has compact support in $M$. Hence, the difference "advanced - retarded solution with source $f$ " defines a linear map

$$
\mathcal{E} \equiv \mathcal{E}^{+}-\mathcal{E}^{-}: C_{0}^{\infty}(M) \longrightarrow S_{0}(M, g)
$$

which associates to each $f \in C_{0}^{\infty}$ the solution $\mathcal{E}^{+} f-\mathcal{E}^{-} f$ of Eq. (2) lying in $S_{0}(M)$. It is not difficult to show (i) that $\mathcal{E}$ is onto, i.e. for each $F \in S_{0}(M)$ there exists a $f \in C_{0}^{\infty}(M)$ for which $F=\mathcal{E} f$, (ii) that $\mathcal{E} f=0$ if and only if there exists a $g \in C_{0}^{\infty}(M)$ such that $f=\left(\nabla^{a} \nabla_{a}-m^{2}\right) g$, and (iii) that the identity

$$
\int_{M} f(x) G(x) d \tau=\sigma(\mathcal{E} f, G)
$$

holds for all $f \in C_{0}^{\infty}(M)$ and for all $G \in S_{0}(M)$. Finally, combining Eqs. (3), (4) and (7) with Eqs. (1), we obtain the identity

$$
[\phi(f), \phi(g)]=i \Delta(f, g), \quad f, g \in C_{0}^{\infty}(M),
$$

where $\Delta$ denotes the two-point distribution [bi-solution of Eq. (2)]

$$
\Delta(f, g) \equiv \sigma(\mathcal{E} f, \mathcal{E} g), \quad f, g \in C_{0}^{\infty}(M)
$$

Equation (8) is the desired expression of the CCR in terms of the smeared field operators $\phi(f)$.

Quantization of the Klein-Gordon field theory will now be complete once we construct the Hilbert space on which $\phi(f)$ act as operators. This can be done directly, by building a Fock space representation of the CCR [Eq. (8)], when a specific "vacuum" state in Hilbert space is singled out by the physics of $(M, g)$. For example, in flat, Minkowski spacetime, the Poincare-invariant Minkowski vacuum can be used to build the standard Fock-space representation of the CCR. However, in a general, globally hyperbolic spacetime, no such preferred vacuum state exists. Furthermore, Fock representations 
of the CCR built from different vacuum states are in general unitarily inequivalent. The only consistent, general way of implementing the CCR into a well-defined mathematical formalism is the algebraic approach. We shall now briefly review the algebraic approach, but only those basics which will be referred to later in the paper. Ref. [2] contains a thorough discussion and ample references; for a more peaceful introduction see [3].

Fundamental to the algebraic approach is the notion of the "Weyl algebra." Given any symplectic vector space $(S, \sigma)$ over $\mathbf{R}$, the pre-Weyl algebra associated to $(S, \sigma)$ is defined as the *-algebra $\mathcal{W}^{\circ}(S, \sigma)$ over the complex field $\mathbf{C}$ consisting of finite sums of the form

$$
\sum_{i} \alpha_{i} W\left(F_{i}\right), \quad \alpha_{i} \in \mathbf{C}, F_{i} \in S,
$$

where the generators $W(F)$ satisfy the relations

$$
\begin{aligned}
W(F) W(G) & =e^{-\frac{i}{2} \sigma(F, G)} W(F+G) \\
& =e^{-i \sigma(F, G)} W(G) W(F)
\end{aligned}
$$

from which the product of two general elements in the form of Eq. (10) can be obtained using linearity and the distributive law. The $*$ operation of $\mathcal{W}^{\circ}(S, \sigma)$ is simply

$$
\left[\sum_{i} \alpha_{i} W\left(F_{i}\right)\right]^{*} \equiv \sum_{i} \overline{\alpha_{i}} W\left(-F_{i}\right)
$$

and the identity element $\mathbf{1}$ is $W(0)$. It is a consequence of the nondegeneracy of the symplectic form $\sigma$ on $S$ that the pre-Weyl algebra $\mathcal{W}^{\circ}(S, \sigma)$ is simple, i.e. it does not contain any proper two-sided ideals [see [4] for a short proof of this]. As a result, there exists a unique $C^{*}$ norm on $\mathcal{W}^{\circ}(S, \sigma)$; the Weyl algebra, $\mathcal{W}(S, \sigma)$, is defined as the $C^{*}$ algebra obtained by completing $\mathcal{W}^{\circ}(S, \sigma)$ under this norm.

Consider now the Weyl algebra $\mathcal{A}$ corresponding to the symplectic space of solutions, $\left(S_{0}, \sigma\right)$, of the Klein-Gordon equation (2), where $\sigma$ is defined by Eq. (4). Using Eq. (7), we can formally rewrite the smeared field operators $\phi(f)$ in the form

$$
\phi(f)=\int_{M} f(x) \phi(x) d \tau=\sigma(\mathcal{E} f, \phi),
$$

which shows that $\phi(f)$ depends only on the solution $\mathcal{E} f$ in $S_{0}(M)$. We can therefore consider the field operators as being smeared with solutions $F \in S_{0}(M)$ instead of with test functions $f \in C_{0}^{\infty}(M)$; hence $\phi(F), F \in S_{0}$, 
denotes the operator $\phi(f)$, where $f \in C_{0}^{\infty}(M)$ is any test function that satisfies $\mathcal{E} f=F$. The generators $W(F)$ of the Weyl algebra $\mathcal{A}$ then correspond, formally, to the exponentiated fields

$$
W(F)=e^{i \phi(F)}=e^{i \phi(f)}, \text { where } F \in S_{0}(M), f \in C_{0}^{\infty}(M), \mathcal{E} f=F
$$

as the reader can verify by comparing the commutation relations (8) with the corresponding relations (11) for $W(F)$. [Note that the CCR in the form of Eq. (8) cannot be represented by an algebra of bounded operators, so in order to to build a $C^{*}$ operator algebra with a well defined norm it is necessary to use the exponentiated version of the CCR given by Eq. (11).] The $C^{*}$ algebra $\mathcal{A}=\mathcal{W}\left(S_{0}, \sigma\right)$ defines the local algebra of field operators for the quantized Klein-Gordon field on $(M, g)$. [The term "local" refers to the existence of the preferred set of closed subalgebras, $\{\mathcal{A}(U)\}$, in $\mathcal{A}$, where for each open subset $U \subset M, \mathcal{A}(U)$ denotes (the closure of) the subalgebra generated by those elements $W(\mathcal{E} f)$ for which $f$ is supported in $U$. This extra structure is of key importance to the generalization of the algebraic approach which we will discuss in the next section.] We will use the more descriptive notation $\mathcal{A}^{(K G, m)}$ for the Klein-Gordon algebra when it is necessary to indicate the explicit field theory it refers to.

All information one might wish to have about the quantized KleinGordon field on $(M, g)$ is contained in the local algebra $\mathcal{A}^{(K G, m)}$. Thus, for example, quantum states of the Klein-Gordon field are nothing but (complex) linear functionals $\omega$ on $\mathcal{A}$ that satisfy the positivity and normalization conditions

$$
\omega\left(x^{*} x\right) \geq 0 \quad \forall x \in \mathcal{A}, \quad \omega(\mathbf{1})=1 .
$$

The $n$-point functions associated to a state $\omega$ can be defined directly in terms of the algebraic structure of $\mathcal{A}$. For example, the two-point function, when it exists, is defined as the distribution [bi-solution of Eq. (2)]

$$
\lambda_{2}(f, g)=-\left.\frac{\partial^{2}}{\partial s \partial t}\right|_{s=t=0}\left[\omega[W(s \mathcal{E} f+t \mathcal{E} g)] e^{-\frac{i}{2} s t \sigma(\mathcal{E} f, \mathcal{E} g)}\right]
$$

acting on test functions $f, g \in C_{0}^{\infty}(M)$. States also provide the connection with Hilbert-space quantization through the so-called "GNS construction" $([4]-[6])$, which associates to each state $\omega$ a Hilbert space, $\left[H_{\omega},<,>\right]$, a "vacuum" vector $\Omega \in H_{\omega}$, and a representation $\rho_{\omega}$ of $\mathcal{A}$ by bounded operators on $H_{\omega}$ such that $\omega(x)=<\Omega, \rho_{\omega}(x) \Omega>\forall x \in \mathcal{A}$. When the two-point function $\lambda_{2}(f, g)$ of $\omega$ exists [Eq. (16)], a one-particle Hilbert space $\mathcal{H}_{\omega}$ can be built from the space of solutions $S_{0}$ using the real part of $\lambda_{2}$ as an inner product; the GNS construction $\left(H_{\omega}, \rho_{\omega}\right)$ is then identical to the standard Fock-space 
construction based on the one-particle Hilbert space $\mathcal{H}_{\omega}$ (see Sect. 3.2 of [2] for details).

The compact, unified way in which the notion of a "quantum state" is formalized is the main advantage of the algebraic approach, and this advantage is present independently of whether the algebra $\mathcal{A}$ has unitarily inequivalent representations (as a concrete algebra of operators on Hilbert space). [A Weyl algebra $\mathcal{W}(S, \sigma)$ has inequivalent representations whenever $S$ is infinite dimensional.] The algebraic viewpoint is in fact indispensable for discussing quantum field theory on any background other than flat, Minkowski spacetime.

\section{A generalization of the algebraic approach}

The quantization formalism outlined above relies in a crucial way on the existence of a global Cauchy surface in spacetime. Globally hyperbolic spacetimes, which are the ones that admit such surfaces, are adequate for discussing most physically interesting problems in General Relativity. Moreover, there exists a rather large collection of arguments, organized around the well-known "cosmic censorship hypothesis," which suggest that under generic conditions global hyperbolicity may in fact be enforced as a consequence of other, more fundamental laws of physics. While still supported by the standard evidence for cosmic censorship, the argument for global hyperbolicity has been challenged increasingly in recent years, and understanding the physics of spacetimes containing closed timelike curves or naked singularities no longer appears to be a question of purely academic interest. Clearly, it is important to remove the global hyperbolicity restriction from the algebraic formulation of quantum field theory.

How can we construct the algebra of the quantum Klein-Gordon field when spacetime lacks a global Cauchy surface $\Sigma$, hence lacks a well-defined symplectic space of solutions $\left(S_{0}, \sigma\right)$ ? Equivalently, how can we formulate quantum field theory in the absence of CCR? The familiar path-integral formalism suggests one way of approaching such a generalization. However, we desire more mathematical clarity and consistency, at least for linear (noninteracting) field theory, than the path-integral approach can provide. For us, the answer lies in widening the notion of "quantum field" so that the construction of the field operators is not tied to the (canonical) quantization of a Hamiltonian system for classical fields. (There is no well defined dynamics for classical fields on a non-globally-hyperbolic spacetime.) In a separate paper submitted elsewhere [7], we report on an extension of the algebraic 
notion of quantum field to general background spaces. The only structure these backgrounds posses is that of a topological space, i.e. they may not even have a spacetime metric defined on them at the outset. The primary motivation for such a drastic generalization is to understand the ultimate origin of spacetime topology, and the motivations for and the consequences of this approach are discussed in detail in [7]. Here we will only summarize the relevant aspects of this generalized algebraic notion of quantum field theory as it applies to the specific problem we are addressing.

Let $X$ be a topological space. A "quantum field theory" on $X$ consists of an abstract $C^{*}$ algebra $\mathcal{A}$ (with identity element $\mathbf{1}$ ), and a map (which we will also denote by $\mathcal{A}$ ) that associates to each open subset $U$ in $X$ a closed subalgebra $\mathcal{A}(U) \subset \mathcal{A}$ such that the following two conditions hold:

(QF1): For every open subset $U \subset X \mathcal{A}(U)$ is a central $C^{*}$ algebra, and $\mathcal{A}(\{\})=\mathrm{C} \cdot \mathbf{1}, \mathcal{A}(X)=\mathcal{A}$.

(QF2): For any collection $\left\{W_{\alpha}\right\}$ of open subsets,

$$
\mathcal{A}\left(\bigcup_{\alpha} W_{\alpha}\right)=\overline{<\bigcup_{\alpha} \mathcal{A}\left(W_{\alpha}\right)>}
$$

Here \{\} denotes the empty set, $\mathbf{C} \cdot \mathbf{1}$ is the $C^{*}$ algebra (isomorphic to the algebra $\mathbf{C}$ of complex numbers) generated by $\mathbf{1}$, a central algebra $\mathcal{B}$ is one with the property that its center,

$$
Z(\mathcal{B}) \equiv\{x \in \mathcal{B} \mid x y=y x \forall y \in \mathcal{B}\}
$$

is equal to $\mathbf{C} \cdot \mathbf{1}[Z(\mathcal{B}) \cong \mathbf{C}],<S>$ denotes the subalgebra generated by a subset $S \subset \mathcal{A}$, and overbar denotes closure. We will call the theory $\mathcal{A}$ "nondegenerate" if $\mathcal{A}(U)$ is strictly bigger than $\mathrm{C}$ for every nonempty open subset $U \subset X$. Note that, in standard quantum field theory, property QF2 does not in general hold for the local algebra of all "observables," but it does hold when $\mathcal{A}$ consists only of (exponentiated) smeared field operators.

Next we incorporate the notion of "locality" into our generalized field theory. For this, let for each point $p \in X C(p)$ denote the set

$$
\begin{aligned}
C(p) \equiv & \{q \in X \mid \nexists \text { open sets } U, V \text { such that } \\
& p \in U, q \in V \text {, and }[\mathcal{A}(U), \mathcal{A}(V)]=0\},
\end{aligned}
$$

where for $A, B \subset \mathcal{A},[A, B]$ denotes the commutator subalgebra generated by elements of the form $\{a b-b a \mid a \in A, b \in B\}$. The set $C(p)$ consists of 
those points $q \in X$ that can "causally communicate" with $p$ through fields in $\mathcal{A}$. [We restrict ourselves throughout to bosonic fields; hence our use of the commutator $[$,$] . It is straightforward to formulate a fermionic version$ of our discussion by replacing commutators with anti-commutators. But note that, in the fermionic case, the generators of the field algebra are the smeared field operators themselves (which are already bounded) instead of the exponentiated fields (14).] Some immediately obvious properties of $C(p)$ are: $q \in C(p)$ iff $p \in C(q), C(p)$ is a closed subset of $X$, and, when $\mathcal{A}$ is nondegenerate, $p \in C(p) \quad \forall p \in X$ (this last result follows from QF1). A continuous curve $\gamma: \mathbf{R} \longrightarrow X$ is called a "connector" if for every $t_{0} \in \mathbf{R}$ there exists an $\epsilon>0$ such that $\gamma(t) \in C[\gamma(s)]$ for all $t, s$ in the interval $\left(t_{0}-\epsilon, t_{0}+\epsilon\right)$. (Thus defined a connector is analogous to a causal curve in spacetime.) The notion of locality for a quantum field theory $\mathcal{A}$ on $X$ is now defined in terms of the topological properties of the sets $C(p)$. Thus, we will say that $\mathcal{A}$ is "weakly local" if the following two conditions are satisfied:

(L): There exists an open neighborhood $V$ around every point $p \in X$ such that for every open neighborhood $U$ of $p$ contained in $V$ the set $U \cap[C(p) \backslash\{p\}]$ is disconnected (here \denotes set difference).

(WL): For all $p \in X C(p)$ is connected.

The theory $\mathcal{A}$ is "strongly local" if it satisfies condition $\mathrm{L}$ and the following stronger version of WL:

(SL): For every $p, q \in X$ such that $q \in C(p)$ there exists a connector $\gamma$ joining $p$ and $q$; in particular, the set $C(p)$ is arcwise connected for all $p \in X$.

It is easy to see that if condition $\mathrm{L}$ is satisfied $\mathcal{A}$ must be nondegenerate.

In physical terms, locality provides for the existence of dynamics, the "finite speed of propagation" of causal signals. Accordingly, condition WL (or SL) guarantees that causal influences propagate from $p$ continuously, and condition L guarantees that signals that communicate with $p$ propagate with "finite speed," and that they connect $p$ to disjoint components of $C(p) \backslash\{p\}$ (which is necessary if dynamics at $p$ is to be determined not only by local evolution equations but also by boundary conditions).

The next notion we will need is that of "isomorphism" between quantum field theories. Let $X$ be a topological space, and let $\mathcal{A}_{1}$ and $\mathcal{A}_{2}$ be field theories on $X$. Then, $\mathcal{A}_{1}$ and $\mathcal{A}_{2}$ are said to be isomorphic, denoted 
$\left(\mathcal{A}_{1}, X\right) \cong\left(\mathcal{A}_{2}, X\right)$, if there is a (isometric) $C^{*}$ isomorphism $\Psi: \mathcal{A}_{1} \longrightarrow \mathcal{A}_{2}$ such that for every open subset $U \subset X$,

$$
\mathcal{A}_{2}(U)=\Psi\left[\mathcal{A}_{1}(U)\right] .
$$

Note that, if $\mathcal{A}$ is an ordinary (e.g. Klein-Gordon) field theory on a globally hyperbolic spacetime $(M, g), h: M \longrightarrow M$ is a diffeomorphism of $M$, and if we define a new theory $h^{*} \mathcal{A}$ on $M$ by (for all open $U \subset M$ )

$$
\left.\left(h^{*} \mathcal{A}\right)(U) \equiv \mathcal{A}^{(K G, m)}\right|_{\left(M, h^{*} g\right)}\left[h^{-1}(U)\right]
$$

[where the field theory that appears on the right-hand side is the standard Klein-Gordon theory corresponding to the spacetime $\left.\left(M, h^{*} g\right)\right]$, then the theories $\mathcal{A}$ and $h^{*} \mathcal{A}$ are isomorphic over $M$. Hence our notion of isomorphism is a generalization of the usual diffeomorphism invariance in curved-spacetime field theory.

We will also need the following result, whose proof can be found (along with a more detailed discussion of the material of this section) in [7]:

Theorem: Let $X$ be a locally compact topological space, and $\mathcal{A}$ a weakly local quantum field theory on $X$. If $U, V \subset X$ are open sets such that $\mathcal{A}(U)$ and $\mathcal{A}(V)$ fail to commute, then there exists a point $p \in U$ and a point $q \in V$ such that $q \in C(p)$.

The generalized Klein-Gordon field theory on a non-globally-hyperbolic spacetime is a field theory in the extended sense we have described in this section. We now turn to the explicit construction of this theory.

\section{Construction of the Klein-Gordon field algebra on an arbitrary spacetime}

Let $(M, g)$ be a spacetime (not necessarily globally hyperbolic). We define a subset $U \subset(M, g)$ to be "locally causal" if $U$ is open, connected, and the spacetime $\left(U,\left.g\right|_{U}\right)$ is globally hyperbolic. [Note that this notion is distinct from the notion of a "globally hyperbolic subset" in $(M, g)$, defined, in the standard way, as a subset $U$ such that (i) strong causality holds on $U$, and (ii) $\forall p$ and $q \in U$ the set $J^{-}(q) \cap J^{+}(p)$ is contained entirely in $U$ and is compact ([8], Chapter 6). In general, a locally causal subset does not satisfy any of these properties; even when the ambient spacetime $(M, g)$ is globally hyperbolic, only (i) and the second half of (ii) hold generally for locally causal $U \subset M$.] Clearly, every spacetime admits an open covering by 
locally causal subsets. We assume that a space of global solutions, $S(M)$, of Eq. (2) has been fixed, through physical considerations, as the configuration space of classical fields on $(M, g)$. Usually, $S(M)$ will simply consist of the space of all $C^{\infty}$ global solutions of the Klein-Gordon equation (2); however, in some cases it may be necessary to include less smooth $\left(C^{k}\right.$ or distributional $)$ solutions in $S(M)$. The resulting Klein-Gordon field theory on $M$ will depend in an essential way on this initial choice of $S(M)$.

Let $T$ denote the real vector space consisting of finite sums of the form

$$
\sum_{i=1}^{n} c_{i}\left(F_{i}, U_{i}\right)
$$

where each $U_{i}$ is a locally causal open subset, $c_{i} \in \mathbf{R}$, and each $F_{i}$ is an element in $S_{0}\left(U_{i}\right)=S_{0}\left(U_{i},\left.g\right|_{U_{i}}\right)$, the space of all $C^{\infty}$ solutions of Eq. (2) defined and compact supported on Cauchy surfaces in $\left(U_{i},\left.g\right|_{U_{i}}\right)$. In $T$ we make the identifications

$$
\begin{aligned}
c(F, U) & =(c F, U), \quad\left(F_{1}, U\right)+\left(F_{2}, U\right)=\left(F_{1}+F_{2}, U\right), \quad(0, U)=0 \\
& \forall U \text { locally causal, } F, F_{1}, F_{2} \in S_{0}(U), c \in \mathbf{R}
\end{aligned}
$$

and only these [thus, in more fancy terms, $T$ is the direct sum $\bigoplus_{U} S_{0}(U)$ where $U$ ranges over all locally causal subsets of $(M, g)$ ]. Let $S^{*}(M)$ denote the algebraic dual of $S(M)$, i.e. the space of all linear functionals on $S(M)$. Each element $x \in T$ defines a linear functional $\alpha_{x} \in S^{*}(M)$ via the relation, $\forall G \in S(M)$,

$$
\alpha_{x}(G) \equiv \sigma_{U}(F, G)=\sigma_{U}\left(F,\left.G\right|_{U}\right) \quad \text { when } x=(F, U)
$$

where $\sigma_{U}$ denotes the Klein-Gordon inner product of the globally hyperbolic spacetime $\left(U,\left.g\right|_{U}\right)$, and the action of $\alpha_{x}$ on $S(M)$ is obtained by linear extension when $x$ is an element of $T$ in the general form Eq. (19). If $U$ and $V$ are locally causal subsets with $U \subset V$, then there exists a natural imbedding $i_{U V}: S_{0}(U) \hookrightarrow S_{0}(V)$ which satisfies

$$
\alpha_{\left(i_{U V} F, V\right)}=\alpha_{(F, U)} \quad \forall F \in S_{0}(U)
$$

This imbedding $i_{U V}$ is constructed as follows: For any $F \in S_{0}(U)$, find a $f \in$ $C_{0}^{\infty}(U)$ such that $F=\mathcal{E}_{U} f$, where $\mathcal{E}_{U} f$ denotes the "advanced minus retarded solution with source $f$ " (see Sect. 1) corresponding to the globally hyperbolic spacetime $\left(U,\left.g\right|_{U}\right)$. Then simply put $i_{U V} F \equiv \mathcal{E}_{V} f$. This construction of $i_{U V} F$ is well defined because $\mathcal{E}_{U} f=0$ iff $f=\left(\nabla^{a} \nabla_{a}-m^{2}\right) g$ for some $g \in$ $C_{0}^{\infty}(U)$, which implies that $\mathcal{E}_{V} f=0$. To prove that Eq. (22) holds, simply 
observe that, using Eq. (7), we can write $\sigma_{U}\left(\mathcal{E}_{U} f, H\right)$ as $\int_{M} f H d \tau$ for all $f \in C_{0}^{\infty}(U)$ and $H \in S(M)$.

It is now possible to define an antisymmetric bilinear form $\Omega$ on the space $T$ by putting

$$
\Omega[(F, U),(G, V)] \equiv \begin{cases}\sigma_{V}\left(i_{U V} F, G\right), & \text { if } U \subset V \\ \sigma_{U}\left(F, i_{V U} G\right), & \text { if } V \subset U \\ 0, & \text { otherwise }\end{cases}
$$

and extending $\Omega$ linearly to all elements of $T$ given in the general form Eq. (19). That $\Omega$ thus defined is a nondegenerate bilinear form on $T$ is easily verified. Hence $(T, \Omega)$ is a symplectic vector space. The Weyl algebra associated to $(T, \Omega)$ might appear, at this point, to be a natural candidate for the generalized Klein-Gordon algebra of $(M, g)$. There are two obvious problems with this, however: First, the space $(T, \Omega)$ is unreasonably big, due clearly to those elements which, from a physical viewpoint, represent the same "solution," but which are contained as distinct vectors in $T$. Second, $(T, \Omega)$ does not reduce to the standard symplectic space $\left[S_{0}(M), \sigma\right]$ (Sect. 1$)$ when $(M, g)$ is globally hyperbolic. We need to somehow shrink $T$ down to the "right" size so that every element it contains represents a distinct local solution. Guidance for doing this is provided by the "principle of self consistency," which says, in essence, that the only physically allowed local solutions of the field equations are those which admit a smooth global extension. (See [9] and [10] for a detailed discussion of this viewpoint in the context of spacetimes with closed timelike curves.) Accordingly, we introduce the subspace $N \subset T$ given by

$$
N=\left\{x \in T \mid \alpha_{x} \equiv 0\right\} .
$$

It is not difficult to verify that $N$ is a symplectic subspace of $T$, i.e. the symplectic form $\Omega$ is nondegenerate when restricted to $N$. The key idea behind the definition Eq. (24) of $N$ is that elements of $T$ which differ by a vector in $N$ are physically equivalent, because their difference cannot be detected using the "local" Klein-Gordon inner products with global solutions. It would now appear that the most natural way to proceed forward from this point is to construct the quotient of the Weyl algebra of $(T, \Omega)$ by the twosided ideal generated by elements $\{W(n) \mid n \in N\}$, i.e. to "mod-out" the unphysical degrees of freedom $W(n)$ from the field algebra. However, as we pointed out in Sect. 1, the Weyl algebra of any symplectic space is simple, i.e. does not admit any nontrivial quotients. In particular, the two-sided ideal generated by $\{W(n) \mid n \in N\}$ in the Weyl algebra of $(T, \Omega)$ is the entire algebra itself. This is a serious technical problem, and the only way to 
circumvent it is to perform the moding-out construction at the level of the vector space $(T, \Omega)$ instead of directly on the Weyl algebra.

So let $Q$ denote the quotient vector space $T / N$. There is a natural symplectic form $\sigma$ on this quotient, namely the "orthogonal projection" of the symplectic form $\Omega$. For simplicity, assume that $T$ admits an orthogonal decomposition

$$
T=N \oplus N^{\perp},
$$

where $N^{\perp} \equiv\{x \in T \mid \Omega(x, n)=0 \quad \forall n \in N\}$. [Note that the decomposition (25) is necessarily a direct sum (provided it exists) since $\Omega$ is nondegenerate on both $T$ and $N$.] Then every $x \in T$ has a unique orthogonal projection $x_{\perp} \in N^{\perp}$ such that $\left(x-x_{\perp}\right) \in N$. Also, for each equivalence class $[x] \in T / N$ and $x \in[x]$, the projection $x_{\perp}$ depends only on the equivalence class $[x]$ and not on the particular representative $x$, i.e. for each $[x] \in Q$ we have a unique projection $x_{\perp} \in N^{\perp}$. The symplectic form $\sigma$ on $T / N$ is now defined simply by

$$
\sigma([x],[y]) \equiv \Omega\left(x_{\perp}, y_{\perp}\right) .
$$

For infinite-dimensional subspaces $N \subset T$ the decomposition (25) does not always exist, however, and a more complicated argument is necessary to construct $\sigma$ in the general case. [When $N$ is finite dimensional, the decomposition (25) clearly exists and can be constructed by purely algebraic means.] We will describe this construction at the very end of this section; for the rest of the section we will assume the construction as given. In fact, throughout the rest of our discussion in Sect. 3 the definition (26) is quite sufficient since for the only concrete example in this section (that of a globally hyperbolic spacetime) the decomposition (25) does exist.

We are now ready to construct the Klein-Gordon field algebra on $(M, g)$. Put

$$
\mathcal{A} \equiv \mathcal{W}(Q, \sigma)
$$

the Weyl algebra corresponding to $(Q, \sigma)$, and let for every open subset $V \subset M$

$$
\begin{aligned}
T(V) & \equiv\left\{x \in T \mid x=\sum_{i}\left(F_{i}, U_{i}\right), \text { where each } U_{i} \subset V, F_{i} \in S_{0}\left(U_{i}\right)\right\} \\
Q(V) & \equiv T(V) / N \\
\mathcal{A}(V) & \equiv \mathcal{W}[Q(V), \sigma] .
\end{aligned}
$$

It is easy to verify the conditions QF1 and QF2 of Sect. 2 for the theory $\mathcal{A}$ defined by Eqs. (28). This is our generalized Klein-Gordon field theory on the spacetime $(M, g)$, and we will continue to denote it by $\mathcal{A}^{(K G, m)}$. 
It may be of interest to note the following heuristic interpretation for the algebra $\mathcal{A}$ : Consider the symbols $W([x])$, where $[x] \in T / N$. We can construct $\mathcal{A}$ as the algebra generated by the symbols $W([x])$ subject to the formal multiplication rule

$$
W([x]) W([y])=\int_{N} \int_{N} W(x+n) W(y+m) V_{\Omega}(d n) V_{\Omega}(d m),
$$

where $W(x)$ are the generators of the Weyl algebra of $(T, \Omega)$, and $x, y \in$ $T$. Here the symbol $V_{\Omega}(d n)$ denotes, formally, the "canonical" symplectic measure on $(N, \Omega)$. More precisely, when $N$ has finite (even) dimension $q$, this measure is given by the $q$-form

$$
V_{\Omega}(d n) \equiv \frac{1}{(4 \pi)^{q / 2}} \Omega \wedge \cdots \wedge \Omega
$$

where the wedge product has $q / 2$ terms. We assume, for the purpose of our heuristic argument, that an infinite-dimensional analogue of $V_{\Omega}(d n)$ also exists. Clearly, the right hand side of the product Eq. (29) depends only on the equivalence classes $[x],[y] \in T / N$. Also, combining Eq. (29) with Eq. (11) yields

$$
\begin{aligned}
W([x]) W([y]) & =\int_{N} \int_{N} e^{-\frac{i}{2} \Omega(x+n, y+m)} W(x+y+n+m) V_{\Omega}(d n) V_{\Omega}(d m) \\
& =W([x+y]) \int_{N} \int_{N} V_{\Omega}(d n) V_{\Omega}(d m) e^{-\frac{i}{2} \Omega(x+n, y+m)}
\end{aligned}
$$

where in the second line we formally identified all $W(x+y+n)$ with $W([x+y])$ for $n \in N$. When $T$ admits the decomposition given by Eq. (25), the complex number multiplying $W([x+y])$ in Eq. (31) can be evaluated easily to be

$$
\begin{aligned}
& e^{-\frac{i}{2} \Omega\left(x_{\perp}, y_{\perp}\right)} \int_{N} \int_{N} V_{\Omega}(d n) V_{\Omega}(d m) e^{-\frac{i}{2} \Omega(n, m)} \\
= & e^{-\frac{i}{2} \Omega\left(x_{\perp}, y_{\perp}\right)}
\end{aligned}
$$

where, using the measure Eq. (30), the last manipulation can be justified rigorously when $N$ is finite dimensional. Thus, it is possible to regard the expression

$$
e^{-\frac{i}{2} \sigma([x],[y])}=\int_{N} \int_{N} V_{\Omega}(d n) V_{\Omega}(d m) e^{-\frac{i}{2} \Omega(x+n, y+m)}
$$

as a formal definition for the symplectic inner product $\sigma$ on the quotient space $Q=T / N$.

We now show that the theory $\mathcal{A}$ just constructed coincides with the ordinary Klein-Gordon field theory (Sect. 1$)$ when $(M, g)$ is globally hyperbolic. 
We take $S(M)$ to be the space of all $C^{\infty}$ global solutions of the Klein-Gordon equation (2) on $(M, g)$. Given $x \in T, x=\sum_{i}\left(F_{i}, U_{i}\right)$, consider the global solutions $F_{i}^{G} \in S_{0}(M)$ defined by

$$
F_{i}^{G} \equiv i_{U_{i} M} F_{i}
$$

[note that, since $(M, g)$ is globally hyperbolic, $M$ itself is a locally causal subset]. We can write

$$
x=\sum_{i}\left(F_{i}, U_{i}\right)=\left[x-\left(\sum_{i} F_{i}^{G}, M\right)\right]+\left(\sum_{i} F_{i}^{G}, M\right) .
$$

It is now easy to verify that the first term in Eq. (35), namely $x-\left(\sum_{i} F_{i}^{G}, M\right)$, is an element of the subset $N$ [Eq. (24)], and that the second term, $\left(\sum_{i} F_{i}^{G}, M\right)$, is orthogonal (under $\Omega$ ) to $N$ [this second term is equivalent, under $\Omega$ inner product with elements of $T$, to the global solution $\sum_{i} F_{i}^{G} \in S(M)$, so the result follows from the definition of $N$ ]. This means we have, in Eq. (35), the orthogonal decomposition Eq. (25) already performed for us. In particular, $x_{\perp}=\left(\sum_{i} F_{i}^{G}, M\right)$. Hence, for $x=\sum_{i}\left(F_{i}, U_{i}\right), y=\sum_{j}\left(H_{j}, V_{j}\right)$,

$$
\begin{aligned}
\sigma([x],[y]) & =\Omega\left(x_{\perp}, y_{\perp}\right) \\
& =\Omega\left[\left(\sum_{i} F_{i}^{G}, M\right),\left(\sum_{j} H_{j}^{G}, M\right)\right] \\
& =\sigma_{M}\left(\sum_{i} F_{i}^{G}, \sum_{j} H_{j}^{G}\right),
\end{aligned}
$$

where $\sigma_{M}$ denotes the global Klein-Gordon inner product of the spacetime $(M, g)$. Therefore we recover the ordinary symplectic space of solutions: $(Q, \sigma) \cong\left(S_{0}, \sigma_{M}\right)$, and the field theory $\mathcal{A}$ defined by Eqs.(28) on a globally hyperbolic spacetime $(M, g)$ is isomorphic to the standard Klein-Gordon theory as constructed in Sect. 1.

The construction we just described for the field algebra of an arbitrary spacetime is quite rigid: once the space of global solutions, $S(M)$, is specified, the local algebra $\mathcal{A}^{(K G, m)}$ is determined completely by the geometry of $(M, g)$. It is clear that for many spacetimes the resulting field theory will not satisfy locality as formulated in Sect. 2. We will call a spacetime $(M, g)$ "microcausal" with respect to the Klein-Gordon field if (i) the local algebra $\mathcal{A}^{(K G, m)}$ defines a strongly local (Sect.2) quantum field theory on $M$, and (ii) every $p \in M$ has a locally causal neighborhood $U$ such that

$$
U \cap C(p) \subset J^{+}(p, U) \cup J^{-}(p, U) .
$$

One important conclusion we can derive from the Theorem stated at the end of Sect. 2 is: if $(M, g)$ is micro-causal and $U, V \subset M$ are spacelike-separated 
open sets, then the subalgebras $\mathcal{A}(U)$ and $\mathcal{A}(V)$ commute. Since otherwise, according to the Theorem in Sect. 2, there would be points $p \in U$ and $q \in V$ such that $q \in C(p)$, and this is in contradiction with the spacelike separation of the open sets $U$ and $V$ because in a micro-causal spacetime any connector is necessarily a causal curve. We will return to a discussion of micro-causality briefly in Sect. 5 after studying some simple examples in the next section.

We turn now, as promised, to the construction of the symplectic form $\sigma$ on $Q=T / N$ in the general case where the decomposition Eq. (25) does not necessarily exist. It will be instructive to first recall the following, more familiar construction: Let $(A,<,>)$ be a (real) inner-product space (not necessarily complete), and $P \subset A$ a closed subspace. Then, on the quotient space $A / P$ there exists a canonical (symmetric, positive-definite) inner product, $<,>_{\perp}$, which can be defined as follows. Given $x \in A$, let $\delta=\inf _{s \in P}\|x-s\|$, where $\|x\|$ denotes the norm $\langle x, x\rangle^{1 / 2}$, and, since $P$ is closed, $\delta>0$ unless $x \in P$. Find a sequence $\left\{x_{k} \in P\right\}$ such that $\left\|x-x_{k}\right\| \rightarrow \delta$. It is easy to show, either from the polarization identity for the norm $\|\cdot\|$, or directly, using the linearity of $P$ and the triangle inequality, that for every $x \in A\left\{x_{k}\right\}$ chosen as above is a Cauchy sequence. Now for any pair of vectors $x, y \in A$ define $<x, y>_{\perp} \equiv \lim _{k \rightarrow \infty}<x-x_{k}, y-y_{k}>$. It can be verified that this limit (which exists since $\left\{\left\langle x-x_{k}, y-y_{k}\right\rangle\right\}$ is a Cauchy sequence in $\mathbf{R}$ ) is independent of the choice of Cauchy sequences $\left\{x_{k}\right\},\left\{y_{k}\right\} \subset P$. Also, it is clear that $\langle x, y\rangle_{\perp}$ thus defined depends only on the equivalence classes $[x],[y] \in A / P$. Hence this construction of $<,\rangle_{\perp}$ gives a well-defined inner product on $A / P$.

Now let $(T, \Omega)$ be a symplectic space. Under the family of seminorms, $\left\{p_{x}(y) \equiv|\Omega(x, y)|, \quad x \in T\right\},(T, \Omega)$ is a locally convex topological vector space ([5], Chapter IV). In this topology, $U$ is an open neighborhood of 0 iff $\forall x \in U$ there exist an $\epsilon>0$ and finitely many $v_{i} \in T$ such that $\left\{y \in T \mid p_{v_{i}}(y-x)<\epsilon\right\} \subset U$. If $N \subset T$ is a closed subspace [our subspace $N$ defined by Eq. (24) is closed], we can define a symplectic form $\sigma$ on $T / N$ by a construction entirely similar to that of $\left\langle,>_{\perp}\right.$ on $A / P$. However, since the topology of $T$ is determined by an uncountable family of seminorms rather than by a single norm, we need to use Cauchy nets instead of Cauchy sequences. Recall that a net is a map from a directed index set into $T$. A directed set is a partially ordered set $\Lambda$ with the property that given any pair $\alpha, \beta \in \Lambda$ there exists a $\gamma \in \Lambda$ such that $\gamma \geq \alpha$ and $\gamma \geq \beta$. A net $\left\{x_{\alpha}\right\}$ is Cauchy if given any open neighborhood $U$ of 0 we can find a $\gamma \in \Lambda$ such that $\left(x_{\alpha}-x_{\beta}\right) \in U$ for all $\alpha, \beta \geq \gamma$, and it converges to $x_{0}$ if for all such $U$ we can find a $\beta \in \Lambda$ such that $\left(x_{\alpha}-x_{0}\right) \in U$ for all $\alpha \geq \beta$. Now fix 
the index set $\Lambda$ to be the directed set consisting of all finite subsets of $T$, partially ordered by inclusion. For each $\alpha \in \Lambda$, let $P_{\alpha}$ denote the seminorm $P_{\alpha}(x) \equiv \sum_{\alpha} p_{v_{\alpha}}(x)$, where the sum is over the (finitely many) vectors $v_{\alpha}$ contained in $\alpha$. Given $x \in T$, we construct a Cauchy net $\left\{x_{\alpha}\right\} \subset N$ as follows. Let for each $\alpha \in \Lambda \delta_{\alpha} \equiv \inf _{n \in N} P_{\alpha}(x-n)$. Pick a vector $x_{\alpha} \in N$ such that $\left|P_{\alpha}\left(x-x_{\alpha}\right)-\delta_{\alpha}\right|<1 /|\alpha|$, where $|\alpha|$ denotes the number of elements in $\alpha \in \Lambda$. It is not difficult to show that the net $\left\{x_{\alpha}\right\}$ thus constructed is Cauchy. Now, for any pair of vectors $x, y \in T$, define $\sigma(x, y) \equiv \lim _{\alpha} \Omega\left(x-x_{\alpha}, y-y_{\alpha}\right)$. This limit exists since the net $\left\{\Omega\left(x-x_{\alpha}, y-y_{\alpha}\right)\right\}$ is Cauchy in $\mathbf{R}$ (hence converges to a unique real number). It can also be verified that the limit is independent of the Cauchy nets $\left\{x_{\alpha}\right\},\left\{y_{\alpha}\right\} \subset N$ chosen, and depends only on the equivalence classes $[x],[y] \in T / N$. Hence $\sigma$ is the desired symplectic structure on $T / N$.

\section{Some simple non-globally-hyperbolic examples}

In this section we will very briefly discuss Klein-Gordon field theory on two examples, both flat, of non-globally-hyperbolic two-dimensional spacetimes. Our discussion is limited to the construction of the local field algebra on these spacetimes in accordance with the general ideas of Sect. 3 .

Our first example is a two-dimensional torus $T^{2}$ with a flat Lorentz metric $\eta$ and a massless Klein-Gordon field. Flat Lorentzian tori admit closed timelike curves through every point. A detailed analysis and classification of these Lorentz tori can be found in Sect. IV of [10]. Here we will work with the simplest such tori: two-dimensional Minkowski spacetime modulo the translation subgroup generated by two orthogonal vectors (generators). We assume, for simplicity, that the ratio of the length of the spacelike generator to the length of the orthogonal timelike one is an integer $p$. When this ratio is irrational, the Lorentz torus does not admit any non-constant global solutions to the massless Klein-Gordon equation, so its field algebra would be trivial $[\mathcal{A}(U)=\mathbf{C} \forall$ open $U \subset M$; i.e. a completely degenerate field theory]. When $p$ is a non-integer but rational number the resulting structure of the field algebra is essentially the same as the integer case. Without loss of generality, we assume that the timelike generator of $\left(T^{2}, \eta\right)$ has length 1 and the spacelike generator length $p$.

We take $S(M)$ to be the space of all $C^{\infty}$ global solutions of $\nabla^{a} \nabla_{a} \phi=0$ on $\left(T^{2}, \eta\right)$. Let $p=1$. Given an element $(F, U) \in T$, we construct the data $\left\{F_{0}, \dot{F}_{0}\right\}$ that $F$ induces on some global spacelike hypersurface of the form $\{t=$ constant $\}$. By Eq. (12) of [10], global solutions $\phi$ are constrained to 
satisfy $\int \dot{\phi}_{0} d s=0$, where $\int d s$ denotes integration over the global $\{t=$ constant $\}$ hypersurface. It is not difficult to show, then, that the symplectic inner product $\sigma$ between two equivalence classes $[(F, U)]$ and $[(G, V)] \in T / N$ is given by

$$
\begin{aligned}
& \sigma([(F, U)],[(G, V)])= \\
& \int\left[F_{0}\left(\dot{G}_{0}-<\dot{G}_{0}>\right)-G_{0}\left(\dot{F}_{0}-<\dot{F}_{0}>\right)\right] d s,
\end{aligned}
$$

where $\langle\cdots\rangle$ denotes the average

$$
<f>\equiv \int f d s
$$

For $p \geq 2$, let $\pi$ denote the finite group of order $p$ consisting of the discrete isometries generated by a unit translation in the $x$-direction, and let for any $f \in C^{\infty}\left(T^{2}\right) \pi f$ denote the function obtained by summing all $p$ images of $f$ under the action of this group. Then the symplectic product $\sigma$ on $T / N$ is

$$
\begin{aligned}
\sigma([(F, U)],[(G, V)]) & = \\
\frac{1}{p} \int\left[\pi F_{0}\left(\pi \dot{G}_{0}-<\pi \dot{G}_{0}>\right)\right. & \left.-\pi G_{0}\left(\pi \dot{F}_{0}-<\pi \dot{F}_{0}>\right)\right] d s,
\end{aligned}
$$

where

$$
<f>\equiv \frac{1}{p} \int f d s
$$

As they stand, the field theories defined by Eqs. (38)-(41) do not satisfy locality. However, as is well known, in two dimensions the presence of infrared divergences in the two-point function makes it necessary to smear a massless scalar field only with those test functions $f \in C_{0}^{\infty}(M)$ that satisfy $\int_{M} f d \tau=0$. This is easily seen to be equivalent to building the space $T$ so that all local solutions $F=\mathcal{E}_{U} f$ satisfy $\left\langle\dot{F}_{0}\right\rangle=0$. With this choice of $T$, the symplectic product $\sigma$ is exactly the same as it would be for the globally hyperbolic cylinder $S^{1} \times \mathbf{R}$, obtained by undoing the identification in the time direction of $\left(T^{2}, \eta\right)$. Therefore, after this modification, the massless Klein-Gordon theory on $\left(T^{2}, \eta\right)$ does satisfy strong locality as formulated in Sect. 2. In fact, in both the cases $p=1$ and $p \geq 2$, and for any $q \in T^{2}$, the union of the two closed null geodesics passing through $q$ constitute the set $C(q)$. Thus micro-causality (Sect. 3) holds for these examples.

Our next example is the two-dimensional spacetime (with timelike singularities) consisting of the open strip $\{0<x<1,-\infty<t<\infty\}$ in Minkowski space (the singularities are at the walls $\{x=0\}$ and $\{x=1\}$ ). 
We let $S(M)$ be the space of all solutions of the (massless or massive) KleinGordon equation which are $C^{\infty}$ on $M$ and $C^{1}$ at the boundaries $\{x=0\}$ and $\{x=1\}$, i.e. we constrain the solutions in $S(M)$ to admit $C^{1}$ extensions (not necessarily as solutions) across these boundaries. [It is possible, but tedious, to show that the field algebra remains exactly the same even when this constraint on the boundary behavior of $S(M)$ is relaxed completely.] Let for each $(F, U) \in T \quad F^{B}$ denote the unique global solution obtained by the following construction: Consider a Cauchy surface $\Sigma$ for $\left(U,\left.\eta\right|_{U}\right)$ and extend it to a global spacelike hypersurface reaching from $x=0$ to $x=1$. The local solution $F$ induces well-posed initial data on this surface which vanish outside of $\Sigma$. Now let $F^{B}$ be the unique solution of the Klein-Gordon equation $\left(\nabla^{a} \nabla_{a}-m^{2}\right) \phi=0$ that evolves from these data with the boundary conditions $\phi=0$ on $\{x=0\}$ and $\phi=0$ on $\{x=1\}$. Then, it is not difficult to show that the symplectic inner product $\sigma$ between two equivalence classes $[(F, U)],[(G, V)] \in T / N$ is given by

$$
\sigma([(F, U)],[(G, V)])=\int\left(F_{0}^{B} \dot{G}_{0}^{B}-G_{0}^{B} \dot{F}_{0}^{B}\right) d s,
$$

where the integral is over any global spacelike hypersurface of the form $\{t=$ constant\} (independence of the surface is insured by the boundary conditions on $F^{B}$ and $\left.G^{B}\right)$. With $T / N$ endowed with this symplectic structure, the field theory $\mathcal{A}^{(K G, m)}$ defined by Eqs. (28) satisfies micro-causality on $(M, \eta)$ for all values of $m$.

\section{Conclusions}

With the general construction we presented in Sect. 3, it now becomes possible to discuss quantum field theory on spacetimes with naked singularities or closed timelike curves in the clean framework of the algebraic approach. For spacetimes with naked singularities, our construction does not eliminate the issue of what boundary conditions are to be imposed on the fields "at" the singularities, but rather delegates this problem to the description of the states of the quantum field instead of the fields themselves. More precisely, although our construction gives an unambiguous description of the local field algebra throughout spacetime, when global hyperbolicity is violated it is no longer sufficient to specify a quantum state only in the vicinity of an initial spacelike hypersurface. Rather, the action of the state (as a linear functional on local smeared field operators) needs to be specified globally on the entire spacetime, a process which in general will involve in-

put that can be interpreted as "boundary conditions" (for the state) at the singularities. For spacetimes with closed timelike curves, our construction naturally leads to a new, microscopic causality condition, "micro-causality," 
that needs to be satisfied for a consistently local (Klein-Gordon) quantum field theory to exist.

The notion of micro-causality is quite distinct from the classical notion of "benignness" introduced earlier in Ref. [10]. Recall ([10]) that, in the present context, a spacetime $(M, g)$ is called benign if every point has an open neighborhood $U$ such that any solution of the Klein-Gordon equation in $U$ which is locally extendible off $U$ (i.e. which does not develop singularities at the boundary of $U$ ) is extendible to a global solution on $(M, g)$. In a benign spacetime, observers making local (classical) measurements of the Klein-Gordon field will be unable to distinguish their spacetime from one which is globally hyperbolic. The spacetimes we discussed in the examples of Sect. 4 are benign with respect to the Klein-Gordon equation (massless for the first example). Benignness and micro-causality are independent properties; i.e. neither of them implies nor is implied by the other. A straightforward quantum-field-theory analogue to the notion of benignness would be the property that (i) micro-causality holds and (ii) every point $p \in M$ has a locally causal neighborhood $U$ with

$$
\left(\left.\mathcal{A}^{(K G, m)}\right|_{U}, U\right) \cong\left(\left.\mathcal{A}^{(K G, m)}\right|_{\left[\left(U,\left.g\right|_{U}\right)\right]}, U\right)
$$

where $\cong$ denotes isomorphism in the quantum-field-theory sense as we discussed in Sect. 2 above, and the field theory on the right hand side denotes the standard Klein-Gordon theory for the globally hyperbolic spacetime $\left(U,\left.g\right|_{U}\right)$. Thus, spacetime is quantum-benign if and only if both strong locality (condition SL) and condition (43) are satisfied. Local measurements of the quantum field operator (and other observables derived from it) in a spacetime satisfying (43) will be inadequate to distinguish that spacetime from one which is globally hyperbolic. The spacetimes discussed in the examples in Sect. 4 are benign also in this quantum sense.

As long as attention is focused on the standard Klein-Gordon or Maxwell field theories, rather than on some generalized version of these where the space of classical solutions is suitably restricted, the micro-causality requirement on spacetimes with closed timelike curves is a much more severe constraint than the classical requirement of benignness. Indeed, it is unlikely, for example, that there exists any four-dimensional compact spacetime which satisfies micro-causality and/or the condition (43) with respect to the KleinGordon field (see [10] for a discussion of classical field theory on compact spacetimes). Uncovering the precise status of micro-causality in spacetimes with closed timelike curves is of obvious importance for understanding the physics of causality violation, and this issue is going to be dealt with in more 
detail in a forthcoming paper $([11])$. 


\section{REFERENCES}

1. J. Dimock, Commun. Math. Phys. 77, 219 (1980); F. G. Friedlander, The Wave Equation in Curved Spacetime (Cambridge University Press, Cambridge, 1973).

2. B. S. Kay and R. M. Wald, Phys. Reports 207, 49 (1991).

3. R. M. Wald, in: Quantum Mechanics in Curved Spacetime, J. Audretsch and V. de Sabbata eds. (Plenum, New York, 1992).

4. B. Simon, in: Mathematics of Contemporary Physics, R. F. Streater ed. (Academic Press, New York, 1972).

5. J. H. Conway, A Course in Functional Analysis (Springer Verlag, New York, 1990).

6. A. Arveson, An Invitation to $C^{*}$ Algebras (Springer Verlag, New York, 1972).

7. U. Yurtsever, The origin of spacetime topology and generalizations of quantum field theory, UCSB Physics Preprint UCSBTH-92-45, (October 1992).

8. S. W. Hawking and G. F. R. Ellis, The large Scale Structure of Spacetime (Cambridge University Press, Cambridge, 1973).

9. J. Friedman, G. Klinkhammer, F. Echeverria, M. S. Morris, I. D. Novikov, K. S. Thorne, and U. Yurtsever, Phys. Rev. D 42, 1915 (1990).

10. U. Yurtsever, J. Math. Phys. 31, 3064 (1990).

11. U. Yurtsever, Quantum theory, locality, and closed timelike curves, UCSB Physics Preprint (in preparation). 